ing of the Commission on the Tectonics of Ore Deposits, which met concurrently with MEGS, described the value of ERTS (Earth resources technology satellite) imagery in a greater understanding of Europe's structure and in the search for mineral resources. Pleas that geologists generally should pay more attention to the special needs of engineers and planners were common, and the need for more mathematical, chemical and experimental approaches to earth science was examined.

European geologists were reminded of their obligation to the rest of the world in more than just an economic sense: G. Jenkins (University of Canterbury, Christchurch, New Zealand) reminded them that stratotypes, the key to the worldwide geological division of time, were often first described in Europe, and that to aid worldwide correlation more must be researched in Europe and published.

A perhaps oversimplified view of the meeting might be that extremely useful summaries of the state of European geology were given, showing that Europe still presents fundamental questions in need of answers. Seeking such answers can occupy European geologists, geochemists and geophysicists for a long time to come. Time, particularly in respect to resources, will not wait for geologists any more than for the rest of mankind, and the participants recognised that closer collaboration between European earth scientists is urgently needed.

About a year ago correspondence in Nature referred to MEGS under the heading 'European earth scientists disunite', suggesting that a schism had opened between European 'geologists' and geophysicists. In this context it is a pity that so few geophysicists attended MEGS for they would have enhanced the meeting. The Reading meeting showed that European earth scientists sincerely desire to act in concert for the benefit of Europe and the world. The UK initiative which created MEGS was warmly appreciated. The establishment of a European Geological Society was discussed and received wide support but no final decision was reached. Instead it was decided to set up an interim committee composed of representatives of a number of the participating countries to promote a MEGS 2 (representatives of both the Netherlands and France indicated a desire to host such a meeting), to cooperate with existing international and European bodies and to encourage closer communication between European earth science societies.
Contrary to widespread belief, although the steady state theory of the Universe may be dead it refuses to lie down, and a small band of enthusiasts continues to try to breathe life into the presumed corpse. But the chances of a miraculous resuscitation do not look too good, as the appearance of two papers together in the same issue of Mon. Not. R. astr. Soc. shows. In one of these contributions (172, 623; 1975) P. K. Das of the Tata Institute develops further the model of QSOs as compact, highly gravitationally redshifted objects that he and J. V. Narlikar put forward recently (Mon. Not. R. astr. Soc. 171, 87; 1975); although Das does not mention the steady state theory, it seems a reasonable assumption that any colleague of Narlikar's who discusses possible non-cosmological contributions to the redshifts of QSOs has the concept not too far towards the back of his mind.

This model has a great deal of interest for theorists and students of relativity, whatever its application in the real Universe, and consists of a static, spherically symmetric distribution of a mass $M$ in a radius $R$ (Das uses dimensional units), in hydrostatic equilibrium, obeying Einstein's equations and divided into two regions, core and envelope. The earlier paper from Das and Narlikar developed equations for the isothermal core-adiabatic stable envelope configuration and discussed some implications for redshifts; in his new work Das concentrates on a discussion of how light propagates from both the surface and interior of such an object, and the effect of this on the angular diameter seen by an external observer.

The observed features of such an object depend on whether $R$ is greater

\section{One step forward and two back for the steady state}

from John Gribbin

than or less than $3 M$, and the ratio $R / M$ is reduced by 'stiffening' either the core or envelope, that is, making the model more relativistic. For light emitted from the surface, as the core or envelope equation of state is stiffened the angular diameter decreases from its Euclidean value; for sufficiently stiff core-envelope combinations, the back of the source becomes visible, with the entire surface eventually being mapped into a thin ring.

But the source need not appear as a ring, and light emitted from the interior can play a part in determining the overall appearance. Combining the effects on light from the core and from the surface with the earlier investigation of redshifts, Das is able to produce an angular diameter-redshift $(\theta(z))$ relationship. He finds that as the core-envelope combination is stiffened a plot of $\theta$ against $z$ shows a minimum, which shifts to higher $z$ as the stiffening increases, and "it is interesting to note that $\theta$ calculated on the basis of cosmological redshift hypotheses also shows a minimum at comparable values of $z$ ".

The properties of this model do depend critically on the equation of state, and for pressure as high as half the energy density the angular dia- meter is constant irrespective of surface area, with multiple imaging taking place. Of course, radiation from the interior will be absorbed by intermediate material en route to the observer, and investigation of the expected absorption feature is the logical next step in developing this intriguing model. Intriguing though it is, however, it is a little artificial, and its relevance to the real world must be in even greater doubt in view of the paper by V. K. Kapahi (ironically, also of the Tata Institute) in the same issue of Mon. Not. R. astr. Soc. (172, 513; 1975). He has looked at angular size counts of extragalactic radio sources, using the $3 \mathrm{CR}$ catalogue, and finds evidence of evolution in source properties even when QSOs are excluded from the sample. This is already difficult to reconcile with steady state ideas, and the same sort of evolution also seems to be present when QSOs are analysed separately, which suggests rather strongly that QSOs really are part of the same family as other extragalactic radio sources.

The best explanation of these variations is to fit them to a simple Einstein-de Sitter cosmology in which the local radio luminosity function (RLF) steepens considerably at high luminosities, the comoving density of high luminosity sources increases with $z$ in a manner similar to that implied by number-luminosity $(\log N-\log S)$ studies and the $V / V_{\mathrm{m}}$ test for QSOs, and mean physical sizes of radio sources evolve with $z$ approximately as $(1+z)^{-1}$. This important confirmation of the pattern suggested by the earlier studies hints strongly that even if a way can be found of mimicking QSO properties in other ways, the cosmological interpretation stands better than ever before. 